\title{
FREE VIBRATION ANALYSIS OF JOINED COMPOSITE CONICAL-CONICAL-CONICAL SHELLS CONTAINING FLUID
}

\author{
Vu Quoc Hien ${ }^{1, *}$, Tran Ich Thinh ${ }^{2}$, Nguyen Manh Cuong ${ }^{2}$, Pham Ngoc Thanh ${ }^{1}$ \\ ${ }^{I}$ Viet Tri University of Industry, Tien Son street, Viet Tri City \\ ${ }^{2}$ Hanoi University of Science and Technology, 1 Dai Co Viet, Hai Ba Trung, Ha Noi \\ "Email: vuquochien47@gmail.com
}

Received: 7 January 2016; Accepted for publication: 20 May 2016

\begin{abstract}
A continuous element (CE) formulation has been presented in this paper for the vibration analysis of three joined cross-ply composite conical shells containing fluid. The three joined cross-ply composite conical shells containing fluid can be considered as the general case for joined conical-cylindrical-conical, joined cylindrical-conical-cylindrical, joined cylindricalconical-conical and joined conical-conical-cylindrical shells containing fluid. Governing equations are obtained using Midlin thick shell theory, taking into account the shear deformation effects. The velocity potential, Bernoulli's equation and impermeability condition have been applied to the shell-fluid interface to obtain an explicit expression for fluid pressure, the dynamic stiffness matrix has been built from which natural frequencies have been calculated. The appropriate expressions among stress resultants and deformations are extracted as continuity conditions at the joining section. A matlab program is coded using the CE formulation. Numerical results on natural frequencies are validated with the available results in other investigations. The effects of the fluid level, semi-vertex angles and lamination sequences on the natural frequencies and circumferential wave number of joined composite conical-conicalconical shells are investigated.
\end{abstract}

Keywords: free vibration, cross-ply composite joined conical-conical-conical shells, dynamics stiffness matrix, continuous element method.

\section{INTRODUCTION}

The joined shells filled with fluid of revolution have many applications in various branches of engineering such as mechanical, aeronautical, marine, civil and power engineering. The research on their mechanical behavior such as vibration characteristics under various external excitations and boundary restrictions has importance in engineering practice. 
The results of investigations on the vibration analysis of cylindrical or conical shells containing fluid and a few publications exist on the vibration analysis of joined cylindrical-conical shells. Different methods for analyzing free vibration of the cylindrical and conical and joined conicalcylindrical shells have been applied. Sivadas and Ganesan [1] investigated the effects of thickness variation on natural frequencies of laminated conical shells by a semi-analytical finite element method. Tong $[2,3]$ proposed the power series expansion approach to study the free vibration of orthotropic and composite laminated conical shells. Shu [4] has employed the differential quadrature method to study the vibration of conical shells. The vibration characteristic for composite cylindrical shells are carried out by using different approaches such as 2D finite element model based on classical thin shell theory [5], 2D analytical method using the cubic spline functions [6], analytical method based on the first-order shear deformation theory (FSDT) [7]. Senthil and Ganesan [8] performed a dynamic analysis on composite conical shells filled with fluid. Kerboua, Lakis and Hmila [9] used a combination of finite element method and classical shell theory to determine the natural frequencies of anisotropic truncated conical shells in interaction with fluid.

Irie et al. [10] used the transfer matrix approach to solve the free vibration of joined isotropic conical-cylindrical shells. Patel et al. [11] presented results for laminated composite joined conical-cylindrical shell with FSDT using finite element method (FEM). Recently, Caresta and Kessissoglou [12] analyzed the free vibrations of joined truncated conicalcylindrical shells, the displacements of the conical sections were solved using a power series solution, while a wave solution was used to describe the displacements of the cylindrical sections, both Donnell-Mushtari and Flugge equations of motion were used. Kouchakazadeh and Shakouri [13] presented study deals with vibrational behavior of two joined cross-ply laminated conical shells, joined cylindrical-conical shells...Governing equations are obtained using thinwalled shallow shell theory of Donnell type and Hamilton's principle, the appropriate expressions among stress resultants and deformations are extracted as continuity condition at the joining section of the cones.

Traditional computational methods like FEM is the discretization operation of the domain which causes errors in dynamic analysis, especially in medium and range frequencies. Numerous Continuous Elements have been established for metal and composite beams [14 - 15] and plates [16]. Nguyen Manh Cuong and Casimir [17] have succeeded in building the DSM for thick isotropic plate and shells of revolution. The continuous element models for composite cylindrical shells and conical shells presented in works of Tran Ich Thinh and Nguyen Manh Cuong [18], [19] imposes a considerable advancement of the study on continuous element method (CEM) for metal and composite structures. Recently, the new research for thick laminated composite joined cylindrical-conical shells by Tran Ich Thinh, Nguyen Manh Cuong and $\mathrm{Vu}$ Quoc Hien [20] has emphasized the CEM in assemblying complex structure.

In this study, the vibrational behavior of a composite joined conical-conical-conical shells containing an incompressible and in viscid liquid was investigated. Illustrative examples are provided to demonstrate the accuracy and efficiency of the developed numerical procedure.

\section{FORMULATION OF JOINED CROSS-PLY COMPOSITE CONICAL-CONICAL- CONICAL SHELLS CONTAINING FLUID}

Let's investigate the joined conical-conical-conical shells containing fluid with $(x, \theta, \mathrm{z})$ coordinates, as shown in Figure 1. Where $x$ is the coordinate long the cones' generators with the origin placed at the middle of the generators, $\theta$ is the circumferential coordinate, and $\mathrm{z}$ is the 
perpendicular to the cones' surfaces. $R_{1}, R_{2}, R_{3}$ and $R_{4}$ are the radius of the system of the cone shells at its first, second, third and the small end, respectively. $\mathrm{L}_{1}, \mathrm{~L}_{2}$ and $\mathrm{L}_{3}$ are lengths of the cone shells respectively. $\alpha_{1}, \alpha_{2}, \alpha_{3}$ are semi-vertex angles of the cone shells. $\mathrm{H}_{1}, \mathrm{H}_{2}, \mathrm{H}_{3}$ are height of the cone shells and $\mathrm{H}$ is height of fluid.

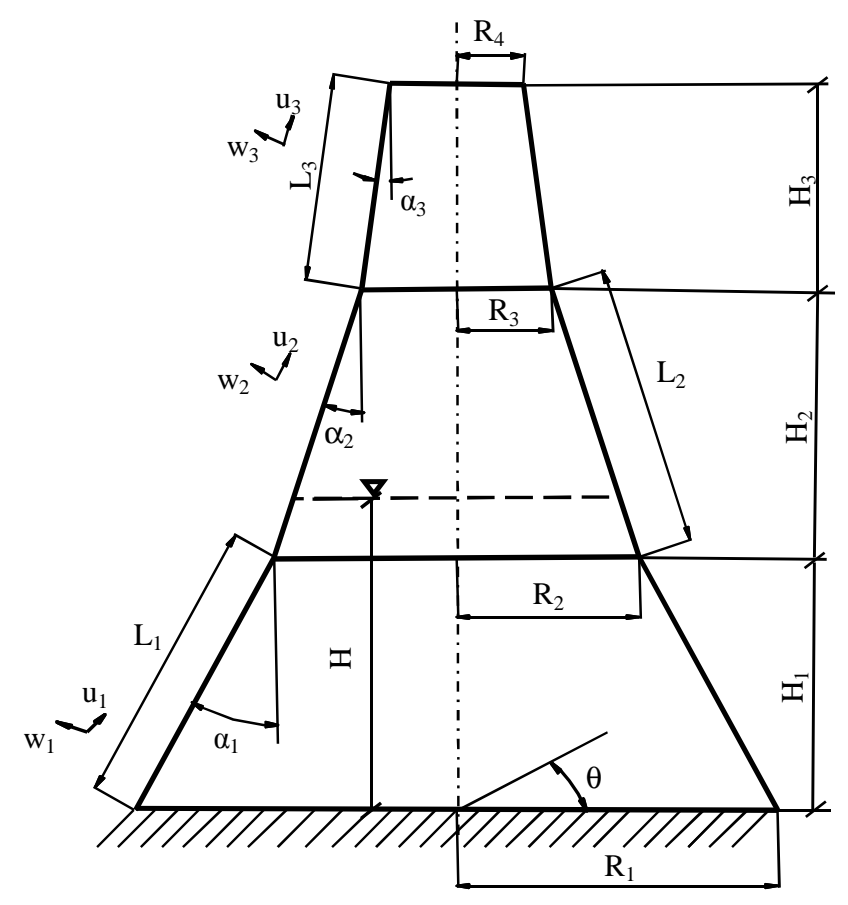

Figure 1.Geometry of joined composite conical- conical -conical shells containing fluid.

\subsection{Composite conical shell containing fluid formulation}

\subsubsection{Constitutive relations}

Consider a laminate composite shell of total thickness $\mathrm{h}$ composed by $\mathrm{N}$ orthotropic layers. The plane stress-reduced stiffnesses are calculated as [23]:

$$
\begin{aligned}
& Q_{11}=\frac{E_{1}}{1-v_{12} v_{21}}, Q_{12}=\frac{v_{12} E_{2}}{1-v_{12} v_{21}}, \\
& Q_{22}=\frac{E_{2}}{1-v_{12} v_{21}}, Q_{66}=G_{12}, Q_{44}=G_{23}, Q_{55}=G_{13}
\end{aligned}
$$

$\mathrm{E}_{\mathrm{i}}, \mathrm{G}_{\mathrm{ij}}, v_{12}, v_{21}$ : elastic constants of the $\mathrm{k}^{\text {th }}$ layer and the laminate stiffness coefficients $\left(A_{i j}, B_{i j}, D_{i j}, F_{i j}\right)$ are defined by:

$$
\begin{aligned}
A_{i j} & =\sum_{k=1}^{N} \bar{Q}_{i j}^{k}\left(z_{k+1}-z_{k}\right), B_{i j}=\frac{1}{2} \sum_{k=1}^{N} \bar{Q}_{i j}^{k}\left(z_{k+1}^{2}-z_{k}^{2}\right), \quad D_{i j}=\frac{1}{3} \sum_{k=1}^{N} \bar{Q}_{i j}^{k}\left(z_{k+1}^{3}-z_{k}^{3}\right)(i, j=1,2,6) \\
F_{i j} & =\sum_{k=1}^{N} Q_{i j}^{k}\left(z_{k+1}-z_{k}\right)(i, j=4,5)
\end{aligned}
$$

where $z_{k-l}$ and $z_{k}$ are the boundaries of the $k^{\text {th }}$ layer. 


\subsubsection{Strains, stress and internal forces resultants}

Following the first-order shear deformation shell theory (FSDT) of Reissner-Mindlin, the displacement components are assumed to be:

$$
u(x, \theta, z, t)=u_{0}(x, \theta, t)+z \varphi_{x}(x, \theta, t) ; v(x, \theta, z, t)=v_{0}(x, \theta, t)+z \varphi_{\theta}(x, \theta, t) ; w(x, \theta, z, t)=w_{0}(x, \theta, t)
$$

The strain-displacement relations of conical shell are (with $R(x)=R_{l}+x \cdot \sin \alpha$ ):

$$
\begin{array}{clrl}
\varepsilon_{x}=\frac{\partial u_{0}}{\partial x} ; & k_{x}=\frac{\partial \varphi_{x}}{\partial x} ; & \varepsilon_{\theta}=\frac{1}{R}\left(u_{0} \sin \alpha+\frac{\partial v_{0}}{\partial \theta}+w_{0} \cos \alpha\right) ; & k_{\theta}=\frac{1}{R}\left(\varphi_{x} \sin \alpha+\frac{\partial \varphi_{\theta}}{\partial \theta}\right) \\
\varepsilon_{x \theta}=\frac{\partial v_{0}}{\partial x}+\frac{1}{R} \frac{\partial u_{0}}{\partial \theta}-\frac{\sin \alpha}{R} v_{0} ; & k_{x \theta}=\frac{1}{R} \frac{\partial \varphi_{x}}{\partial \theta}+\frac{\partial \varphi_{\theta}}{\partial x}-\frac{\sin \alpha}{R} \varphi_{\theta} ; & \gamma_{\theta Z}=\frac{-\cos \alpha}{R} v_{0}+\frac{1}{R} \frac{\partial w_{0}}{\partial \theta}+\varphi_{\theta}
\end{array}
$$

The force and moment resultants are expressed in terms of strains for cross-ply laminated composite conical shell as follows:

$$
\left[\begin{array}{l}
\mathrm{N} \\
\mathrm{M} \\
\mathrm{Q}
\end{array}\right]=\left[\begin{array}{lll}
A & B & 0 \\
B & D & 0 \\
0 & 0 & F
\end{array}\right]\left[\begin{array}{l}
\varepsilon \\
k \\
\gamma
\end{array}\right]
$$

Substituting equations (2) and (4) in equations (5), the force-displacement relation expressions for laminated composite conical shell are written as follows:

$$
\begin{gathered}
N_{x}=A_{11} \frac{\partial u_{0}}{\partial x}+\frac{A_{12}}{R}\left(u_{0} \sin \alpha+\frac{\partial v_{0}}{\partial \theta}+w_{0} \cos \alpha\right)+B_{1} \frac{\partial \varphi_{x}}{\partial x}+\frac{B_{12}}{R}\left(\varphi_{x} \sin \alpha+\frac{\partial \varphi_{\theta}}{\partial \theta}\right) ; \\
N_{\theta}=A_{12} \frac{\partial u_{0}}{\partial x}+\frac{A_{22}}{R}\left(u_{0} \sin \alpha+\frac{\partial v_{0}}{\partial \theta}+w_{0} \cos \alpha\right)+B_{12} \frac{\partial \varphi_{x}}{\partial x}+\frac{B_{22}}{R}\left(\varphi_{x} \sin \alpha+\frac{\partial \varphi_{\theta}}{\partial \theta}\right) ; \\
N_{x \theta}=A_{66}\left(\frac{\partial v_{0}}{\partial x}+\frac{1}{R} \frac{\partial u_{0}}{\partial \theta}-\frac{\sin \alpha}{R} v_{0}\right)+B_{66}\left(\frac{1}{R} \frac{\partial \varphi_{x}}{\partial \theta}+\frac{\partial \varphi_{\theta}}{\partial x}-\frac{\sin \alpha}{R} \varphi_{\theta}\right) ; \\
M_{x}=B_{11} \frac{\partial u_{0}}{\partial x}+\frac{B_{12}}{R}\left(u_{0} \sin \alpha+\frac{\partial v_{0}}{\partial \theta}+\frac{w_{0} \cos \alpha}{R}\right)+D_{1} \frac{\partial \varphi_{x}}{\partial x}+\frac{D_{22}}{R}\left(\varphi_{x} \sin \alpha+\frac{\partial \varphi_{\theta}}{\partial \theta}\right) ; \\
M_{\theta}=B_{12} \frac{\partial u_{0}}{\partial x}+\frac{B_{22}}{R}\left(u_{0} \sin \alpha+\frac{\partial v_{0}}{\partial \theta}+w_{6} \cos \alpha\right)+D_{2} \frac{\partial \varphi_{x}}{\partial x}+\frac{D_{22}}{R}\left(\varphi_{x} \sin \alpha+\frac{\partial \varphi_{\theta}}{\partial \theta}\right) ; \\
Q_{x}=k F_{55}\left(\frac{\partial w_{0}}{\partial x}+\varphi_{x}\right) ; \\
M_{66}\left(\frac{\partial v_{0}}{\partial x}+\frac{\partial u_{0}}{R \partial \theta}-\frac{\sin \alpha}{R} v_{0}\right)+D_{66}\left(\frac{1}{R} \frac{\partial \varphi_{x}}{\partial \theta}+\frac{\partial \varphi_{\theta}}{\partial x}-\frac{\sin \alpha}{R} \varphi_{\theta}\right) ; \\
Q_{\theta}=k F_{44}\left(\frac{-\cos \alpha}{R} v_{0}+\frac{1}{R} \frac{\partial w_{0}}{\partial \theta}+\varphi_{\theta}\right)
\end{gathered}
$$

where $k$ is the shear correction factor $(k=5 / 6)$

\subsubsection{Equations of motion}

The equations of motion using the FSDT for laminated composite conical shell containing fluid are:

$$
\begin{aligned}
& \frac{\partial N_{x}}{\partial x}+\frac{\sin \alpha}{R}\left(N_{x}-N_{\theta}\right)+\frac{1}{R} \frac{\partial N_{x \theta}}{\partial \theta}=I_{0} \ddot{u}_{0}+I_{1} \ddot{\varphi}_{x} ; \frac{\partial N_{x \theta}}{\partial x}+\frac{2 \sin \alpha}{R} N_{x \theta}+\frac{1}{R} \frac{\partial N_{\theta}}{\partial \theta}+\frac{\cos \alpha}{R} Q_{\theta}=I_{0} \ddot{v}_{0}+I_{1} \ddot{\varphi}_{\theta} \\
& \frac{\partial M_{x}}{\partial x}+\frac{\sin \alpha}{R}\left(M_{x}-M_{\theta}\right)+\frac{1}{R} \frac{\partial M_{x \theta}}{\partial \theta}-Q_{x}=I_{1} \ddot{u}_{0}+I_{2} \ddot{\varphi}_{x} ; \frac{\partial M_{x \theta}}{\partial x}+\frac{2 \sin \alpha}{R} M_{x \theta}+\frac{1}{R} \frac{\partial M_{\theta}}{\partial \theta}-Q_{\theta}=I_{1} \ddot{v}_{0}+I_{2} \ddot{\varphi}_{\theta} \\
& \frac{\partial Q_{x}}{\partial x}+\frac{1}{R} \frac{\partial Q_{\theta}}{\partial \theta}+\frac{\sin \alpha}{R} Q_{x}-\frac{\cos \alpha}{R} N_{\theta}-P \cos \alpha=I_{0} \ddot{w}_{0}
\end{aligned}
$$

where $u_{0}, v_{0}, w_{0}$ : the displacements at the mid-surface; $\varphi_{x}, \varphi_{\theta}$ : the rotations of tangents along the $x$ and $\theta$ axes; $P$ : hydrodynamic pressure acting on the shell surface. 
and:

$$
I_{i}=\sum_{k=1}^{N} \int_{z_{k}}^{z_{k+1}} \rho^{(k)} z^{i} d z \quad(\mathrm{i}=0,1,2)
$$

with $\rho^{(\mathrm{k})}$ is the material mass density of the $k^{\text {th }}$ layer.

\subsection{Fluid equations}

The potential function $\Phi(z, \theta, x, t)$ satisfies the Laplace equation in cylindrical coordinates $(z, \theta, x)$ :

$$
\frac{\partial^{2} \Phi}{\partial z^{2}}+\frac{1}{z} \frac{\partial \Phi}{\partial z}+\frac{1}{z^{2}} \frac{\partial^{2} \Phi}{\partial \theta^{2}}+\frac{\partial^{2} \Phi}{\partial x^{2}}=0
$$

Then, the Bernoulli equation is written:

$$
\frac{\partial \Phi}{\partial t}+\frac{P}{\rho_{f}}=0
$$

By linearizing this expression, the pressures on the internal regions are:

$$
P=-\left.\rho \frac{\partial \Phi}{\partial t}\right|_{\Sigma}
$$

The condition of impermeability of the surface of shell in contact with fluid can be expressed as:

$$
v_{f}=\left.\frac{\partial \Phi}{\partial z}\right|_{\Sigma}=\left.\frac{\partial w_{0}}{\partial t}\right|_{\Sigma}
$$

where $w$ is the normal displacement of the shell, $v_{\mathrm{f}}$ is the velocity of fluid.

The hydrodynamic pressure acting on the cylindrical shell is then defined by [20]:

$$
P=-\rho_{f} \frac{1}{m+k_{n} R I_{m+1}\left(k_{n} R\right) / I_{m}\left(k_{n} R\right)} \frac{\partial^{2} w_{0}}{\partial t^{2}}=m^{*} \frac{\partial^{2} w_{0}}{\partial t^{2}}
$$

where:

$$
m^{*}=-\rho_{f} \frac{1}{m+k_{n} R I_{m+1}\left(k_{n} R\right) / I_{m}\left(k_{n} R\right)}
$$

This value will be introduced in (7) in order to establish the Dynamic Stiffness Matrix for the studied structure.

\subsection{Continuity conditions}

The continuity conditions at the conical- conical shell joint can be obtained from Kouchakazadeh and Shakouri [13] as follows:

$$
\begin{aligned}
& u_{i} \cos \alpha_{i}-w_{i} \sin \alpha_{i}=u_{i+1} \cos \alpha_{i+1}-w_{i+1} \sin \alpha_{i+1} ; u_{i} \sin \alpha_{i}+w_{i} \cos \alpha_{i}=u_{i+1} \sin \alpha_{i+1}+w_{i+1} \cos \alpha_{i+1} \\
& v_{i}=v_{i+1} ; \quad \quad \frac{\partial w_{i}}{\partial x_{i}}=\frac{\partial w_{i+1}}{\partial x_{i+1}} ; \quad M_{x \theta}^{i}=M_{x \theta}^{i+1} ; \quad M_{x}^{i}=M_{x}^{i+1} \\
& N_{x}^{i} \cos \alpha_{i}-Q_{x}^{i} \sin \alpha_{i}=N_{x}^{i+1} \cos \alpha_{i+1}-Q_{x}^{i+1} \sin \alpha_{i+1} ; \quad N_{x}^{i} \sin \alpha_{i}+Q_{x}^{i} \cos \alpha_{i}=N_{x}^{i+1} \sin \alpha_{i+1}+Q_{x}^{i+1} \cos \alpha_{i+1}
\end{aligned}
$$


where: $i=1,2$.

\section{CONTINUOUS ELEMENT FORMULATION FOR CROSS-PLY COMPOSITE JOINED CONICAL-CONICAL-CONICAL SHELLS CONTAINING FLUID}

\subsection{Strong formulation}

Here, the state-vector $\mathbf{y}=\left\{u_{0}, v_{0}, w_{0}, \varphi_{x}, \varphi_{\theta} N_{x}, N_{x \theta}, Q_{x}, M_{x}, M_{x \theta}\right\}^{T}$. Next, the Lévy series expansion for state variables is written as:

$$
\begin{gathered}
\left\{u_{o}(x, \theta, t), w_{o}(x, \theta, t), \varphi_{\theta}(x, \theta, t), N_{x}(x, \theta, t), Q_{x}(x, \theta, t), M_{x}(x, \theta, t)\right\}^{T}=\sum_{m=1}^{\infty}\left\{u_{m}(x), w_{m}(x), \varphi_{\theta m}(x), N_{x_{m}}(x), Q_{x m}(x), M_{x_{m}}(x)\right\}^{T} \cos m \theta^{i \omega t} \\
\left\{v_{o}(x, \theta, t), \varphi_{x}(x, \theta, t), N_{x \theta}(x, \theta, t), M_{x \theta}(x, \theta, t)\right\}^{T}=\sum_{m=1}^{\infty}\left\{v_{m}(x), \varphi_{x m}(x), N_{x \theta m}(x), M_{x \theta m}(x)\right\}^{T} \sin m \theta e^{i \omega t}
\end{gathered}
$$

where $m$ is the number of circumferential wave.

Substituting (14) in equations (6) and (7), the ordinary differential equations in the $x$ coordinate for the $m^{\text {th }}$ mode can be expressed in the matrix form for each circumferential mode $m$ as [18-22]:

$$
\begin{aligned}
& \frac{d u_{m}}{d x}=c_{4} \sin \alpha \cdot u_{m}+m c_{4} v_{m}+c_{4} \cos \alpha \cdot w_{m}+c_{5} \sin \alpha \cdot \varphi_{x m}+m c_{5} \varphi_{\theta m}+\frac{D_{11}}{c_{1}} N_{x m}-\frac{B_{11}}{c_{1}} M_{x m} \\
& \frac{d v_{m}}{d x}=\frac{m}{R} u_{m}-\frac{\sin \alpha}{R} v_{m}-\frac{D_{66}}{c_{10}} N_{x \theta m}+\frac{B_{66}}{c_{10}} M_{x \theta m} \\
& \frac{d w_{m}}{d x}=-\varphi_{x m}+\frac{1}{k F_{55}} Q_{x m} \\
& \frac{d \varphi_{x m}}{d x}=c_{2} \sin \alpha \cdot u_{m}+m c_{2} v_{m}+c_{2} \cos \alpha \cdot w_{m}+c_{3} \sin \alpha \cdot \varphi_{x m}+m c_{3} \varphi_{\theta m}-\frac{B_{11}}{c_{1}} N_{x m}+\frac{A_{11}}{c_{1}} M_{x m} \\
& \frac{d \varphi_{\theta m}}{d x}=\frac{m}{R} \varphi_{x m}-\frac{\sin \alpha}{R} \varphi_{\theta m}+\frac{B_{66}}{c_{10}} N_{x \theta m}-\frac{A_{66}}{c_{10}} M_{x \theta m} \\
& \frac{d N_{x m}}{d x}=\left(c_{6} \sin { }^{2} \alpha-I_{0} \varpi^{2}\right) u_{m}+m c_{6} \sin \alpha \cdot v_{m}+c_{6} \sin \alpha \cos \alpha \cdot w_{m}+\left(c_{7} \sin ^{2} \alpha-I_{1} \omega^{2}\right) \varphi_{x m} \\
& +m c_{7} \sin \alpha \cdot \varphi_{\theta m}-\sin \alpha\left(c_{4}+\frac{1}{R}\right) N_{x m}-\frac{m}{R} N_{x \theta m}-c_{2} \sin \alpha \cdot M_{x m} \\
& \frac{d N_{x \theta m}}{d x}=m c_{6} \sin \alpha \cdot u_{m}+\left(m^{2} c_{6}+\frac{k F_{44} \cos { }^{2} \alpha}{R^{2}}-I_{0} \omega^{2}\right) v_{m}+m \cos \alpha\left(c_{6}+\frac{k F_{44}}{R^{2}}\right) w_{m}+ \\
& +m c_{7} \sin \alpha \cdot \varphi_{x m}+\left(m^{2} c_{7}-\frac{k F_{44} \cos \alpha}{R}-I_{1} \omega^{2}\right) \varphi_{\theta m}-m c_{4} N_{x m}-\frac{2 \sin \alpha}{R} N_{x \theta m}-m c_{2} M_{x m}
\end{aligned}
$$




$$
\begin{aligned}
& \frac{d Q_{x m}}{d x}=c_{6} \sin \alpha \cos \alpha \cdot u_{m}+m \cos \alpha\left(c_{6}+\frac{k F_{44}}{R^{2}}\right) v_{m}+\left(c_{6} \cos ^{2} \alpha+\frac{m^{2} k F_{44}}{R^{2}}-I_{0} \omega^{2}-m^{*} \omega^{2}\right) w_{m} \\
& +c_{7} \sin \alpha \cos \alpha \cdot \varphi_{x m}+\left(m c_{7} \cos \alpha-\frac{m k F_{44}}{R}\right) \varphi_{\theta m}-c_{4} \cos \alpha \cdot N_{x m}-\frac{\sin \alpha}{R} Q_{x m}-c_{2} \cos \alpha \cdot M_{x m} \\
& \frac{d M_{x m}}{d x}=\left(2 c_{8} \sin ^{2} \alpha-I_{1} \omega^{2}\right) \cdot u_{m}+2 m c_{8} \sin \alpha \cdot v_{m}+2 c_{8} \sin \alpha \cos \alpha \cdot w_{m}++\left(2 c_{9} \sin ^{2} \alpha-I_{2} \omega^{2}\right) \cdot \varphi_{x m} \\
& +2 m c_{9} \sin \alpha \cdot \varphi_{\theta m}-2 c_{5} \sin \alpha \cdot N_{x m}+Q_{x}-\left[2 \sin \alpha\left(c_{3}+\frac{1}{R}\right)\right] M_{x m}-\frac{m}{R} M_{x \theta m} \\
& \frac{d M_{x \theta m}}{d x}=m c_{8} \sin \alpha \cdot u_{m}+\left(m^{2} c_{8}-\frac{k F_{44} \cos \alpha}{R}-I_{1} \omega^{2}\right) v_{m}+m\left(c_{8} \cos \alpha-\frac{k F_{44}}{R}\right) w_{m}+ \\
& +m c_{9} \sin \alpha \cdot \varphi_{x m}+\left(m^{2} c_{9}+k F_{44}-I_{2} \omega^{2}\right) \varphi_{\theta m}-m c_{5} \cdot N_{x m}-m c_{3} M_{x m}-\frac{2 \sin \alpha}{R} \cdot M_{x \theta m}
\end{aligned}
$$

with:

$$
\begin{gathered}
c_{1}=A_{11} D_{11}-B_{11}^{2}, c_{2}=\left(A_{12} B_{11}-A_{11} B_{12}\right) / R c_{1}, c_{3}=\left(B_{11} B_{12}-A_{11} D_{12}\right) / R c_{1}, \\
c_{4}=\left(B_{11} B_{12}-A_{12} D_{11}\right) / R c_{1} ; c_{5}=\left(B_{11} D_{12}-B_{12} D_{11}\right) / R c_{1} ; \\
c_{6}=\left(A_{12} c_{4}+B_{12} c_{2}+A_{22} / R\right) / R ; c_{7}=\left(A_{12} c_{5}+B_{12} c_{3}+B_{22} / R\right) / R ; \\
c_{8}=\left(B_{12} c_{4}+D_{12} c_{2}+B_{22} / R\right) / R ; c_{9}=\left(B_{12} c_{5}+D_{12} c_{3}+D_{22} / R\right) / R ; \\
c_{10}=B_{66}^{2}-A_{66} D_{66} \\
\frac{d \mathbf{y}_{m}}{d x}=\mathbf{A}_{m} \mathbf{y}_{m} \quad \text { with } \mathbf{A}_{m} \text { is a } 10 x 10 \text { matrix }
\end{gathered}
$$

\subsection{Dynamic transfer matrix, dynamic stiffness matrix $K(\omega)$}

The dynamic transfer matrix $[\mathbf{T}]_{\mathrm{m}}$ is given by:

$$
T_{m}(\omega)=e^{\int_{0}^{L} A_{m}(x, \omega) d x}
$$

Then $[\mathbf{T}]_{\mathrm{m}}$ is separated into four blocks:

$$
[\mathbf{T}]_{m}=\left[\begin{array}{ll}
\mathbf{T}_{11} & \mathbf{T}_{12} \\
\mathbf{T}_{21} & \mathbf{T}_{22}
\end{array}\right]
$$

Finally, the dynamic stiffness matrix $[\mathbf{K}(\omega)]_{\mathrm{m}}$ for conical shell containing fluid is determined by:

$$
[\mathbf{K}(\omega)]_{m}=\left[\begin{array}{cc}
\mathbf{T}_{12}^{-1} \mathbf{T}_{11} & -\mathbf{T}_{12}^{-1} \\
\mathbf{T}_{21}-\mathbf{T}_{22} \mathbf{T}_{12}^{-1} \mathbf{T}_{11} & \mathbf{T}_{22} \mathbf{T}_{12}^{-1}
\end{array}\right]_{m}
$$

The assembly procedure of the finite element method is used to construct the Dynamic Stiffness Matrix for combined cylindrical-conical-conical shells containing fluid. 


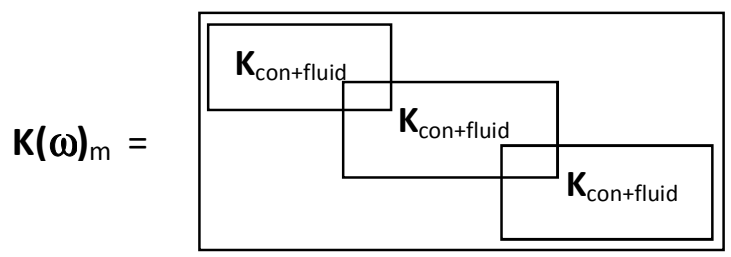

Natural frequencies will be extracted from the harmonic responses of the structure by using the procedure detailed in [18 - 22].

\section{NUMERICAL RESULTS AND DISCUSSION}

\subsection{Comparative study}

A computer program based on Matlab is developed using CEM of composite joined conical-conical-conical shells containing different fluid level.

Lowest frequency parameters $\Omega=\omega R_{l}\left(\rho h / A_{11}\right)^{1 / 2}$ are validated with analytical solutions of Kouchakzadeh [13] for a free-clamped (FC) joined cross-ply laminated conical-conical shells in Table 1: $\mathrm{L} / \mathrm{R}_{3}=1 ; \mathrm{h} / \mathrm{R}_{3}=0.01 ; \mathrm{h}=2 \mathrm{~mm} ; \mathrm{L}_{1}=\mathrm{L}_{2}=\mathrm{L} ; \mathrm{L}_{3}=0 ; \alpha_{1}=60^{\circ}, \alpha_{2}=30^{\circ}$ and $\alpha_{1}=30^{\circ}$, $\alpha_{2}=60^{\circ} ; \mathrm{E}_{1}=135 \mathrm{GPa} ; \mathrm{E}_{2}=8.8 \mathrm{GPa} ; \mathrm{G}_{12}=4.47 \mathrm{GPa} ; v_{12}=0.33 ; \rho=1600 \mathrm{~kg} / \mathrm{m}^{3}$. The results of present study are in good agreement with Kouchakzadeh's results.

Table 1. Comparison of lowest frequency parameter of joined cross-ply laminated conical-conical shells for various types lamination sequences and cone angles (FC boundary conditions).

\begin{tabular}{ccccc}
\hline No & $\begin{array}{c}\text { Layers/ } \\
\text { cone angles } \alpha_{1}=60^{0} ; \alpha_{2}=30^{0}\end{array}$ & Kouchakzadeh $[13]$ & CEM & $\begin{array}{c}\text { Errors }(\%) \\
\text { CEM-[13] }\end{array}$ \\
\hline 1 & {$[0,90]$} & $0.0339(4)$ & $\mathbf{0 . 0 3 4 1}$ & $\mathbf{0 . 5 9}$ \\
2 & {$[90,0]$} & $0.0338(4)$ & $\mathbf{0 . 0 3 4 0}$ & $\mathbf{0 . 5 9}$ \\
3 & {$[0,0,0]$} & $0.0231(4)$ & $\mathbf{0 . 0 2 3 1}$ & $\mathbf{0}$ \\
4 & {$[0,90,0]$} & $0.0302(4)$ & $\mathbf{0 . 0 3 0 3}$ & $\mathbf{0 . 3 3}$ \\
5 & {$[0,0,90]$} & $0.0294(4)$ & $\mathbf{0 . 0 2 9 5}$ & $\mathbf{0 . 3 4}$ \\
6 & {$[0,90,90]$} & $0.0447(4)$ & $\mathbf{0 . 0 4 4 7}$ & $\mathbf{0}$ \\
7 & {$[90,90,0]$} & $0.0454(4)$ & $\mathbf{0 . 0 4 5 3}$ & $\mathbf{0 . 2 2}$ \\
8 & {$[90,90,90]$} & $0.1303(3)$ & $\mathbf{0 . 1 3 1 3}$ & $\mathbf{0 . 7 6}$ \\
9 & {$[0,90]_{2}$} & $0.0426(4)$ & $\mathbf{0 . 0 4 2 7}$ & $\mathbf{0 . 2 3}$ \\
10 & {$[0,90]_{\mathrm{s}}$} & $0.0367(4)$ & $\mathbf{0 . 0 3 6 9}$ & $\mathbf{0 . 5 4}$ \\
11 & {$[90,0]_{2}$} & $0.0426(4)$ & $\mathbf{0 . 0 4 2 8}$ & $\mathbf{0 . 4 6}$ \\
12 & {$[90,0]_{\mathrm{s}}$} & $0.0476(3)$ & $\mathbf{0 . 0 4 7 7}$ & $\mathbf{0 . 2 1}$ \\
\hline 1 & {$[0,90]$} & $0.0274(4)$ & $\mathbf{0 . 0 2 8 1}$ & $\mathbf{2 . 4 9}$ \\
2 & {$[90,0]$} & $0.0273(4)$ & $\mathbf{0 . 0 2 8 1}$ & $\mathbf{2 . 8 5}$ \\
3 & {$[0,0,0]$} & $0.0152(4)$ & $\mathbf{0 . 0 1 5 7}$ & $\mathbf{3 . 1 8}$ \\
4 & {$[0,90,0]$} & $0.0210(4)$ & $\mathbf{0 . 0 2 1 6}$ & $\mathbf{2 . 7 8}$ \\
5 & {$[0,0,90]$} & $0.0227(4)$ & $\mathbf{0 . 0 2 3 3}$ & $\mathbf{2 . 5 8}$ \\
\hline
\end{tabular}




\begin{tabular}{ccccc}
\hline 6 & {$[0,90,90]$} & $0.0378(4)$ & $\mathbf{0 . 0 3 8 2}$ & $\mathbf{1 . 0 5}$ \\
7 & {$[90,90,0]$} & $0.0380(3)$ & $\mathbf{0 . 0 3 8 1}$ & $\mathbf{0 . 2 6}$ \\
8 & {$[90,90,90]$} & $0.0999(3)$ & $\mathbf{0 . 1 0 1 0}$ & $\mathbf{1 . 0 9}$ \\
9 & {$[0,90]_{2}$} & $0.0336(3)$ & $\mathbf{0 . 0 3 3 9}$ & $\mathbf{0 . 8 8}$ \\
10 & {$[0,90]_{\mathrm{S}}$} & $0.0273(4)$ & $\mathbf{0 . 0 2 7 9}$ & $\mathbf{2 . 1 5}$ \\
11 & {$[90,0]_{2}$} & $0.0332(3)$ & $\mathbf{0 . 0 3 3 5}$ & $\mathbf{0 . 9 0}$ \\
12 & {$[90,0]_{\mathrm{S}}$} & $0.0368(3)$ & $\mathbf{0 . 0 3 7 1}$ & $\mathbf{0 . 8 1}$ \\
\hline
\end{tabular}

\subsection{Results and discussion}

The above formulation is used to compute natural frequencies for a Free-clamped joined cross-ply laminated composite conical-conical-conical shells containing fluid: $L / R_{4}=1$; $\mathrm{h} / \mathrm{R}_{4}=0.01 ; \mathrm{h}=2 \mathrm{~mm} ; \mathrm{L}_{1}=\mathrm{L}_{2}=\mathrm{L}_{3}=\mathrm{L} ; \alpha_{1}=60^{\circ}, \alpha_{2}=45^{0}, \alpha_{3}=30^{\circ} ; \mathrm{E}_{1}=135 \mathrm{GPa} ; \mathrm{E}_{2}=8.8 \mathrm{GPa}$; $\mathrm{G}_{12}=4.47 \mathrm{GPa} ; v_{12}=0.33 ; \rho=1600 \mathrm{~kg} / \mathrm{m}^{3}$; layers $\left[0^{\circ} / 90^{0}\right],\left[0^{\circ} / 90^{\circ} / 0^{0}\right],\left[0^{0} / 90^{0} / 0^{0} / 90^{0}\right]$. The effects of fluid level and the number of layers on fundamental frequencies of free-clamped laminated composite joined conical-conical-conical shells containing fluid are illustrated in Table 2.

Table 2. The fundamental frequency $\omega(\mathrm{Hz})$ of joined cross-ply laminated conical-conical-conical shells containing fluid, $n=1$ (FC boundary conditions).

\begin{tabular}{|c|c|c|c|c|c|}
\hline $\begin{array}{l}\text { Fluid } \\
\text { Level }\end{array}$ & $\mathrm{m}$ & $\begin{array}{c}\text { Configuration } \\
{[0 / 90]}\end{array}$ & $\begin{array}{c}\text { Configuration } \\
{[0 / 90 / 0]}\end{array}$ & $\begin{array}{c}\text { Configuration } \\
{[0 / 90 / 0 / 90]}\end{array}$ & $\begin{array}{c}\% \text { Reduction } \\
\text { with respect to empty } \\
{[0 / 90 / 0 / 90] \text { shell }}\end{array}$ \\
\hline \multirow{5}{*}{$\mathrm{H}=0$} & 1 & 595.1 & 576.4 & 597.7 & - \\
\hline & 2 & 395.9 & 396.2 & 400.0 & - \\
\hline & 3 & 261.0 & 273.0 & 271.0 & - \\
\hline & 4 & 208.6 & 247.6 & 237.2 & - \\
\hline & 5 & 226.7 & 219.3 & 294.0 & - \\
\hline \multirow{5}{*}{$\mathrm{H}=0.5 \mathrm{H}_{1}$} & 1 & 507.0 & 512.7 & 525.0 & 12.16 \\
\hline & 2 & 358.5 & 366.1 & 365.8 & 8.55 \\
\hline & 3 & 246.2 & 259.5 & 256.0 & 5.54 \\
\hline & 4 & 200.7 & 236.8 & 225.8 & 4.81 \\
\hline & 5 & 222.3 & 217.9 & 273.7 & 6.90 \\
\hline \multirow{5}{*}{$\mathrm{H}=\mathrm{H}_{1}$} & 1 & 412.0 & 387.6 & 414.1 & 30.72 \\
\hline & 2 & 271.3 & 269.4 & 273.7 & 31.58 \\
\hline & 3 & 191.1 & 198.7 & 197.1 & 27.27 \\
\hline & 4 & 162.4 & 185.4 & 177.7 & 25.08 \\
\hline & 5 & 190.9 & 202.3 & 213.3 & 27.45 \\
\hline \multirow{5}{*}{$\mathrm{H}=\mathrm{H}_{1}+0.5 \mathrm{H}_{2}$} & 1 & 289.6 & 272.6 & 290.5 & 51.40 \\
\hline & 2 & 188.5 & 187.1 & 189.8 & $\mathbf{5 2 . 5 5}$ \\
\hline & 3 & 141.5 & 146.6 & 145.5 & 46.31 \\
\hline & 4 & 126.9 & 143.3 & 137.5 & 42.03 \\
\hline & 5 & 151.7 & 167.9 & 166.0 & 43.54 \\
\hline \multirow{5}{*}{$\mathrm{H}=\mathrm{H}_{1}+\mathrm{H}_{2}$} & 1 & 273.0 & 259.6 & 273.8 & 54.19 \\
\hline & 2 & 183.9 & 182.8 & 185.0 & 53.75 \\
\hline & 3 & 137.1 & 143.0 & 141.5 & 47.79 \\
\hline & 4 & 103.7 & 120.7 & 115.7 & 51.22 \\
\hline & 5 & 130.4 & 134.7 & 148.8 & 49.39 \\
\hline
\end{tabular}




\begin{tabular}{cccccc}
\hline & 1 & 219.8 & 211.4 & 220.5 & $\mathbf{6 3 . 1 1}$ \\
$\mathrm{H}=\mathrm{H}_{1}+\mathrm{H}_{2}+0.5 \mathrm{H}_{3}$ & 2 & 155.9 & 154.9 & 156.6 & $\mathbf{6 0 . 8 5}$ \\
& 3 & 105.1 & 111.3 & 109.4 & $\mathbf{5 9 . 6 3}$ \\
& 5 & 76.4 & 89.6 & 86.0 & $\mathbf{6 3 . 7 4}$ \\
& 5 & 104.6 & 107.2 & 121.0 & $\mathbf{5 8 . 8 4}$ \\
\hline $\mathrm{H}=\mathrm{H}_{1}+\mathrm{H}_{2}+\mathrm{H}_{3}$ & 2 & 223.7 & 216.8 & 224.4 & $\mathbf{6 2 . 4 6}$ \\
(fully) & 3 & 93.7 & 152.3 & 154.0 & $\mathbf{6 1 . 5 0}$ \\
& 4 & 72.5 & 98.3 & 97.6 & $\mathbf{6 3 . 9 9}$ \\
& 5 & 78.0 & 74.1 & 83.4 & $\mathbf{6 4 . 8 4}$ \\
\hline
\end{tabular}

Next, natural frequencies are calculated for a Free-clamped joined cross-ply laminated composite conical-conical-conical shells containing fluid: $L / R_{4}=1 ; \mathrm{h} / \mathrm{R}_{4}=0.01 ; \mathrm{h}=2 \mathrm{~mm}$; $\mathrm{L}_{1}=\mathrm{L}_{2}=\mathrm{L}_{3}=\mathrm{L} ; \alpha_{1}=45^{0}, \alpha_{2}=30^{0}, \alpha_{3}=15^{0} ; \mathrm{E}_{1}=135 \mathrm{GPa} ; \mathrm{E}_{2}=8.8 \mathrm{GPa} ; \mathrm{G}_{12}=4.47 \mathrm{GPa} ; v_{12}=$ $0.33 ; \rho=1600 \mathrm{~kg} / \mathrm{m}^{3} ;$ layers $\left[0^{0} / 90^{0}\right],\left[0^{0} / 90^{\circ} / 0^{0}\right],\left[0^{0} / 90^{\circ} / 0^{0} / 90^{0}\right]$. The effects of cone angles on fundamental frequencies of free-clamped laminated composite joined conical-conical-conical shells containing fluid are illustrated in Tables 2-3.

Table 3. The fundamental frequency $\omega(\mathrm{Hz})$ for joined cross-ply laminated conical-conical-conical shells containing fluid, $n=1$.

\begin{tabular}{|c|c|c|c|c|c|}
\hline $\begin{array}{l}\text { Fluid } \\
\text { Level }\end{array}$ & $\mathrm{m}$ & $\begin{array}{c}\text { Configuration } \\
{[0 / 90]}\end{array}$ & $\begin{array}{c}\text { Configuration } \\
{[0 / 90 / 0]}\end{array}$ & $\begin{array}{c}\text { Configuration } \\
{[0 / 90 / 0 / 90]}\end{array}$ & $\begin{array}{c}\% \text { Reduction } \\
\text { with respect to empty } \\
{[0 / 90 / 0 / 90] \text { shell }}\end{array}$ \\
\hline \multirow{5}{*}{$\mathrm{H}=0$} & 1 & 787.4 & 780.9 & 787.8 & - \\
\hline & 2 & 520.7 & 525.2 & 526.6 & - \\
\hline & 3 & 328.5 & 337.8 & 338.6 & - \\
\hline & 4 & 249.0 & 283.2 & 286.9 & - \\
\hline & 5 & 245.8 & 227.1 & 327.3 & - \\
\hline \multirow{5}{*}{$\mathrm{H}=0.5 \mathrm{H}_{1}$} & 1 & 620.9 & 609.7 & 635.4 & 19.35 \\
\hline & 2 & 438.9 & 447.0 & 446.6 & 15.19 \\
\hline & 3 & 298.4 & 310.0 & 307.9 & 9.07 \\
\hline & 4 & 237.4 & 270.7 & 267.4 & 6.80 \\
\hline & 5 & 241.8 & 225.8 & 300.6 & 8.16 \\
\hline \multirow{5}{*}{$\mathrm{H}=\mathrm{H}_{1}$} & 1 & 499.1 & 470.4 & 500.3 & 36.49 \\
\hline & 2 & 310.5 & 306.9 & 312.2 & 40.71 \\
\hline & 3 & 214.5 & 218.5 & 219.1 & 35.29 \\
\hline & 4 & 179.4 & 195.1 & 193.1 & 32.69 \\
\hline & 5 & 201.6 & 205.6 & 220.1 & 32.75 \\
\hline \multirow{5}{*}{$\mathrm{H}=\mathrm{H}_{1}+0.5 \mathrm{H}_{2}$} & 1 & 342.2 & 324.7 & 342.7 & 56.50 \\
\hline & 2 & 210.6 & 208.6 & 211.4 & 59.86 \\
\hline & 3 & 155.0 & 157.6 & 157.9 & $\mathbf{5 3 . 3 7}$ \\
\hline & 4 & 136.4 & 147.0 & 145.4 & 49.32 \\
\hline & 5 & 157.8 & 166.8 & 169.0 & 48.37 \\
\hline
\end{tabular}




\begin{tabular}{cccccc}
\hline & 1 & 322.1 & 271.7 & 322.4 & $\mathbf{5 9 . 0 8}$ \\
$\mathrm{H}=\mathrm{H}_{1}+\mathrm{H}_{2}$ & 2 & 204.9 & 186.6 & 205.5 & $\mathbf{6 0 . 9 8}$ \\
& 3 & 150.2 & 156.6 & 153.6 & $\mathbf{5 4 . 6 4}$ \\
& 5 & 110.1 & 111.3 & 121.9 & $\mathbf{5 7 . 5 1}$ \\
& 5 & 136.9 & 122.7 & 154.7 & $\mathbf{5 2 . 7 3}$ \\
\hline $\mathrm{H}=\mathrm{H}_{1}+\mathrm{H}_{2}+0.5 \mathrm{H}_{3}$ & 3 & 116.1 & 120.8 & 119.9 & $\mathbf{6 7 . 2 3}$ \\
& 2 & 174.0 & 173.4 & 174.4 & $\mathbf{6 6 . 8 8}$ \\
& 4 & 83.9 & 93.8 & 93.5 & $\mathbf{6 4 . 5 9}$ \\
& 5 & 114.0 & 111.9 & 131.7 & $\mathbf{6 7 . 4 1}$ \\
$\mathrm{H}=\mathrm{H}_{1}+\mathrm{H}_{2}+\mathrm{H}_{3}$ & 2 & 168.2 & 169.3 & 257.3 \\
(fill-fluid) & 3 & 102.4 & 105.4 & 170.2 & $\mathbf{6 7 . 3 5}$ \\
& 4 & 76.4 & 86.2 & 88.1 & $\mathbf{6 7 . 6 8}$ \\
& 5 & 78.2 & 72.1 & 104.0 & $\mathbf{6 8 . 7 8}$ \\
\hline
\end{tabular}

The effects of fluid level, cone angles and the number of layers on fundamental frequencies of free-clamped laminated composite joined conical-conical-conical shells containing fluid are illustrated by the Tables 2-3 and Figures 2-3.

The result show that, natural frequency of composite joined conical-conical-conical shells containing fluid reduces as fluid level increases, filled fluid can reduce significantly the natural frequency of a laminated composite joined conical-conical-conical shells containing fluid. Natural frequency of composite joined conical-conical-conical shells containing fluid reduces when the cone angles increase. Increase number of layers in constant thickness, increases natural frequency of composite joined conical-conical-conical shells containing fluid. In addition, the use of $90^{\circ}$ layers as outer ones increase the rigidity of the structure and results in higher values of frequency.

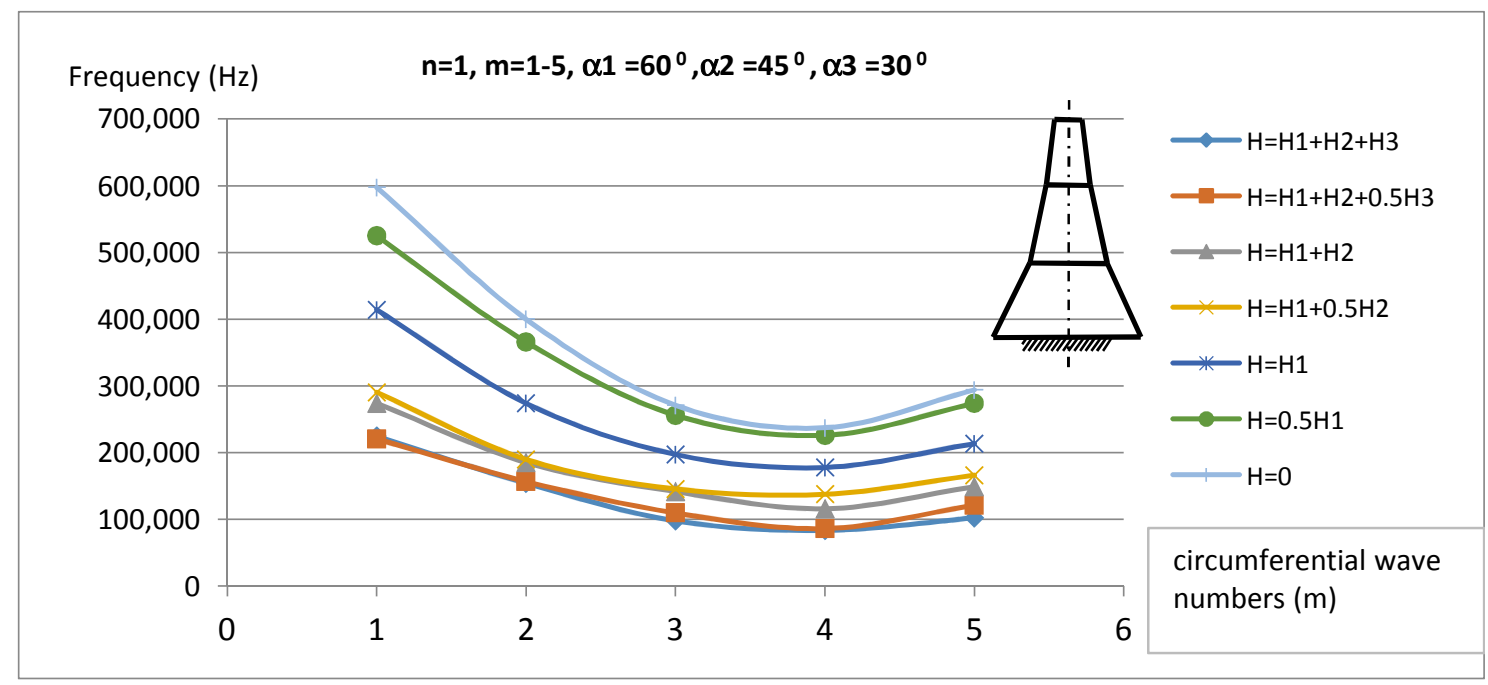

Figure 2. Effect of fluid level on fundamental frequencies of free-clamped laminated composite joined conical-conical-conical shells containing fluid. 


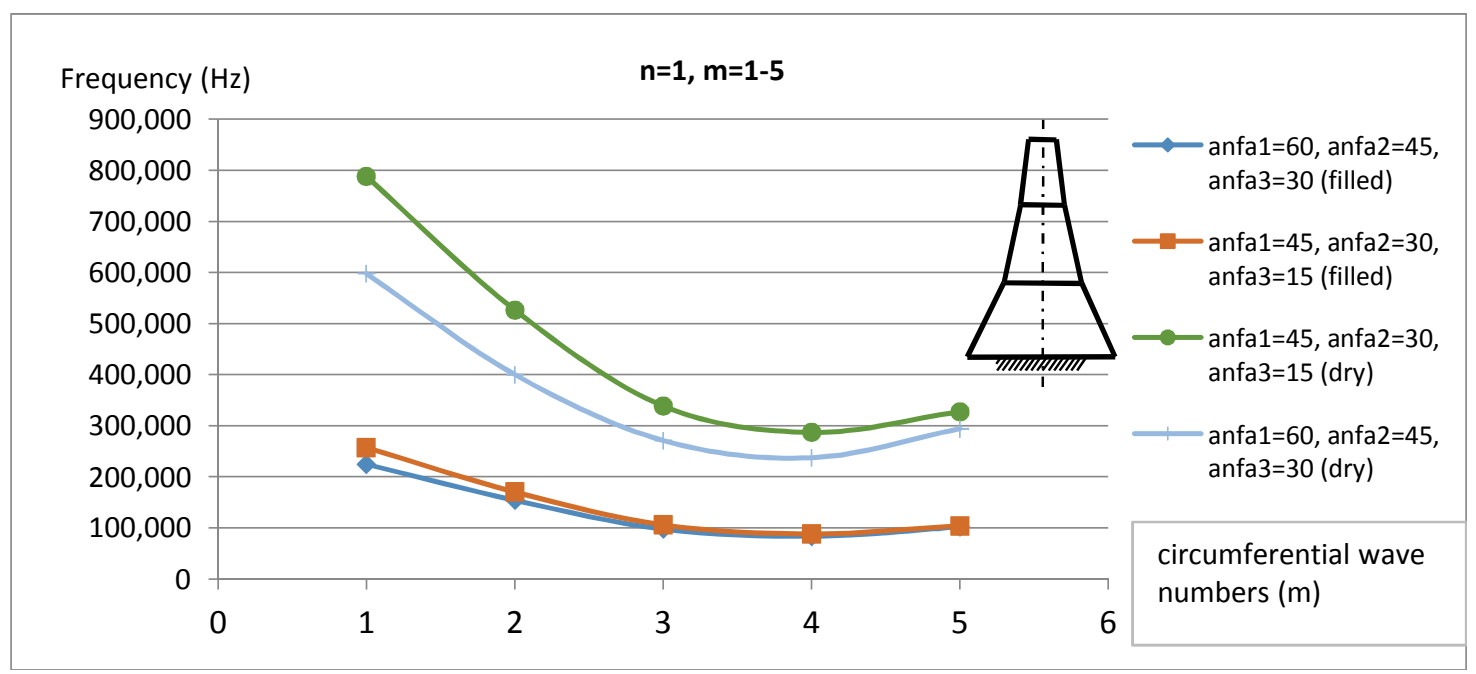

Figure 3. Effect of cone angles on fundamental frequencies of free-clamped laminated composite joinedconical-conical-conical shells containing fluid.

\section{CONCLUSIONS}

Based on the numerical results presented in this paper, the following conclusions may be drawn:

Continuous Element Method can be used to calculate natural frequencies of thick joined cross-ply laminated joined conical-conical-conical shells containing fluid.

The filled fluid can reduce significantly the natural frequencies of thick joined cross-ply laminated joined conical-conical-conical shells.

Natural frequency of composite joined conical-conical-conical shells containing fluid reduces as the cone angles increase.

Natural frequency of composite joined conical-conical-conical shells containing fluid increases when number of layers increase.

The present Continuous Element model can be expanded to solve the vibration problem of joined composite conical-conical-conical shell containing fluid on foundations.

Acknowledgements: This research is funded by Vietnam National Foundation for Science and Technology Development (NAFOSTED) under grant number: 107.02-2013.25.

\section{REFERENCES}

1. Sivadas K. R., Ganesan N. - Vibration analysis of thick composite clamped conical shells of varying thickness, Journal of Sound and Vibration 152 (1992) 27-37.

2. Tong L. Y. - Free vibration of orthotropic conical shells, International Journal of Engineering Science 31 (1993) 719-733.

3. Tong L. Y. - Free vibration of composite laminated conical shells, International Journal of Mechanical Sciences 35 (1993) 47-61. 
4. Shu C. - An efficient approach for free vibration analysis of conical shells, International Journal of Mechanical Sciences 38 (1996) 935-949.

5. Narita Y., Ohta Y., Saito M. - Finite element study for natural frequencies of cross-ply laminated cylindrical shells, Composite structures 26 (1993) 55-62.

6. Viswanathan K. K., Kim K. S., Lee J. H., Koh H. S., and Lee J. B. - Free vibration of multi-layered circular cylindrical shell with cross-ply walls, including shear deformation by using spline function method, Journal of Mechanical Science and Technology 22 (2008) 2062-2075.

7. Khdeir A. A., Reddy J. N., and Fredrick D. - Astudy of bending, vibration and buckling of cross-ply circular cylindrical shells with variuos shells theories, Int. J. Eng. Sci. 27 (11) (1989) 1337-1351.

8. Senthil Kumar D., Ganesan N. - Dynamic analysis of conical shells conveying fluid, Journal of Sound and Vibration 310 (2008) 38-57.

9. Kerboua Y., Lakis A. A., Hmila M. - Vibration analysis of truncated conical shells subjected to flowing fluid, Applied Mathematical Modeling 34 (2010) 791-809.

10. Irie, Yamara and Muramoto - Free vibration of joined conical-cylindrical shells, Journal of Sound and Vibration 95 (1984) 31-39.

11. Patel, Ganapathi and Kamat - Free vibration characteristics of laminated composite joined conical-cylindrical shells, Journal of Sound and Vibration 237 (2000) 920-930.

12. Caresta and Kessissoglou - Free vibration characteristics of isotropic coupled cylindricalconical shells, Journal of Sound and Vibration 329 (2010) 733-751.

13. Kouchakazadeh and Shakouri - Free vibration analysis of joined cross-ply laminated conical shells, International Journal of Mechanical Sciences 78 (2014) 118-125.

14. Banerjee J. R., Sobey A. J. - Dynamic stiffness formulation and free vibration analysis of a three-layered sandwich beam, Int J Solids Struct. 42 (2005) 2181-97.

15. Banerjee J. R.,Williams F. W. - Coupled bending-torsional dynamic stiffness matrix for Timoshenko beam elements, Comput Struct. 42 (1992) 301-10.

16. Nguyen Manh Cuong - Eléments Continus de plaques et coques avec prise en compte du cisaillement transverse, Application à l'interaction fluide-structure, Thèse de Doctorat, Université Paris VI (2003).

17. Casimir J. B., Nguyen Manh Cuong - Thick shells of revolution: Derivation of the dynamic stiffness matrix of continuous elements and application to a tested cylinder, Computers \& structures 85 (23-24) (2007) 1845-1857.

18. Tran Ich Thinh, Nguyen Manh Cuong - Dynamic stiffness matrix of continuous element for vibration of thick cross-ply laminated composite cylindrical shells, Compos Struct. 98 (2013) 93-102.

19. Nguyen Manh Cuong, Tran Ich Thinh, Ta Thi Hien. Vibration analysis of thick laminated composite conical shells by CEM, Tuyển tập HNKHTQ lần Thứ IX, Hanoi (2012).

20. Tran Ich Thinh, Nguyen Manh Cuong, Vu Quoc Hien - Dynamic Stiffness Method for free vibration analysis of partial fluid-filled orthotropic circular cylindrical shells, Vietnam Journal of Mechanics 37 (1) (2015) 29-42.

21. Nguyen Manh Cuong, Tran Ich Thinh and Vu Quoc Hien - Vibration analysis of cross-ply composite joined conical-cylindrical shells by Continuous Element Method, Proceedings 
of the International Conference on Engineering Mechanics and Automation - ICEMA3 (2014) 401-408.

22. Xi Z. C., Yam L. H., and Leung T. P. - Free vibration of laminated composite circular cylindrical shell partially filled with fluid, Composite Part B 28B (1997) 359-375.

23. Reddy J. N. - Mechanics of laminated Composite Plates and Shells Theory and Analysis, CRC Press (2004).

\begin{tabular}{|c|c|c|c|c|c|c|c|c|c|}
\hline \multicolumn{10}{|c|}{ Appendix: Matrix $[A(\omega)]_{10 \times 10}$ : } \\
\hline$c_{4} \sin \alpha$ & $m q$ & $c_{4} \cos x$ & $c_{5} \sin \alpha$ & $m \xi$ & $\frac{D_{1}}{c}$ & 0 & 0 & $\frac{B_{1}}{c}$ & 0 \\
\hline$\frac{m}{R}$ & $\frac{\sin \alpha}{R}$ & 0 & 0 & 0 & 0 & $\frac{D_{66}}{c_{00}}$ & 0 & 0 & $\frac{B_{66}}{c_{00}}$ \\
\hline 0 & 0 & 0 & -1 & 0 & 0 & 0 & $\frac{1}{k F}$ & 0 & 0 \\
\hline$c_{2} \sin \alpha$ & $m \varepsilon$ & $c_{2} \cos x$ & $c_{3} \sin \alpha$ & $m_{\xi}$ & $\frac{B_{1}}{c_{1}}$ & 0 & 0 & $\frac{A_{1}}{c_{1}}$ & 0 \\
\hline 0 & 0 & 0 & $\frac{m}{R}$ & $-\frac{\sin \alpha}{R}$ & 0 & $\frac{B_{66}}{c_{10}}$ & 0 & 0 & $-\frac{A_{66}}{c_{10}}$ \\
\hline$\left(c_{6} \sin \alpha-I_{0} \omega^{3}\right)$ & $m c ̧ \sin \alpha$ & $c_{6} \sin \alpha \cos x$ & $\left(c_{7} \sin ^{2} \alpha-I_{1} \omega^{2}\right)$ & $m g q \sin \alpha$ & $\left(c_{4}+\frac{1}{R}\right) \sin \alpha$ & $-\frac{m}{R}$ & 0 & $-c_{2} \sin \alpha$ & 0 \\
\hline$m q \sin \alpha$ & $\left(m^{2} c_{6}+\frac{k F_{44} \cos \alpha}{R^{2}}-I_{0} \omega\right)$ & $m \cos \left(c_{6}+\frac{k F_{44}}{R^{2}}\right)$ & $m g \sin \alpha$ & $\left(m^{2} c_{7}-\frac{k F_{44} \cos \alpha}{R}-I_{1} \omega\right)$ & $-m_{q}$ & $\frac{2 \sin \alpha}{R}$ & 0 & $-m \varepsilon$ & 0 \\
\hline$c_{6} \sin x \cos x$ & $m \cos \left(c_{6}+\frac{k F_{4}}{R^{2}}\right)$ & $\left(c_{6} \cos ^{2} \alpha+\frac{m^{2} k F_{44}}{R^{2}}-I_{0} \omega^{2}-m^{*} \omega^{2}\right)$ & $c_{7} \sin \alpha \cos \alpha$ & $m\left(G_{7} \cos x-\frac{k F_{44}}{R}\right)$ & $-c_{4} \cos x$ & 0 & $\frac{\sin \alpha}{R}$ & $-c_{2} \cos x$ & 0 \\
\hline$\left(2 \varepsilon_{8} \sin ^{2} \alpha-I_{1} \omega^{2}\right)$ & $2 m \epsilon_{8} \sin \alpha$ & $2 c_{8} \sin \alpha \cos \alpha$ & $\left(2 c_{9} \sin ^{2} \alpha-I_{2} \omega^{2}\right)$ & $2 m_{\S} \sin \alpha$ & $-2 c_{5} \sin \alpha$ & 0 & 1 & $-2 \sin \left(c_{3}+\frac{1}{R}\right)$ & $\frac{m}{R}$ \\
\hline$m_{\xi} \sin \alpha$ & $\left(m^{2} c_{8} \frac{k F_{44} \cos \alpha}{R}-I_{1} \omega\right)$ & $\eta\left(c_{8} \cos \alpha \frac{k F_{4}}{R}\right)$ & $m g ̧ \sin \alpha$ & $\left(m^{2} c_{9}+k F_{44}-I_{2} \omega^{3}\right)$ & $-m \xi$ & 0 & 0 & $-m c ̧$ & $\frac{2 \sin \alpha}{R}$ \\
\hline
\end{tabular}

